\title{
Código Francês de Deontologia MÉdica: COMENTÁRIo CRÍTICO ${ }^{(1)}$
}

FRENCH CODE OF MEDICAL ETHICS: CRITICAL ANALYSIS

Joël Moret-Bailly*

\section{RESUMO:}

Este artigo reflete sobre a evolução da regulamentação ética da profissão médica na França, por meio da análise dos diversos códigos deontológicos franceses ao longo dos séculos XIX e XX, culminando na edição da lei de 4 de março de 2002 que trata dos direitos dos pacientes à informação. O manuscrito discute a constituição jurídica dos códigos deontológicos, as questões tratadas pela deontologia e os valores e concepções profissionais que nela se refletem.

\section{Palavras-chave:}

Código Deontológico; França; Medicina.

\section{ABSTRACT:}

This article reflects on the evolution of ethical regulation of medical profession in France, through the analysis of the various French ethical codes over the nineteenth and twentieth centuries, culminating in the issue of the law of March $4^{\text {th }} .2002$ concerning to the rights of patients to information. The paper discusses the development of legal ethical codes, the questions approached by the ethics and the professional values and concepts which are reflected in it.

(*) Professor de Direito Privado e Ciências Criminais, Université de Lyon, Université Jean Monnet. Saint-Étienne/França. E-mail: j.moret.bailly @gmail.com.

Recebido em 27.10.11. Aprovado em 30.11.11

(1) Nota do editor: a íntegra do Código Francês de Deontologia Médica está disponível em < http:// www.conseil-national.medecin.fr/groupe/17/tous $>$. 


\section{Keywords:}

Ethical Code; France; Medicine.

\section{INTRODUÇÃO}

A reflexão relativa à deontologia médica nasceu, na França, em meados do século XIX, inspirada na organização profissional das atividades relacionadas à justiça e ao direito (advogados, notários, oficiais de justiça etc.) do início dos anos 1810.

Nesse contexto o termo "deontologia" retoma uma palavra criada em 1834 pelo filósofo utilitarista inglês Jeremy Bentham ${ }^{(2)}$. O filósofo explica que "a palavra Deontologia deriva de duas palavras gregas, deon-ontos (o que é adequado) e logos (conhecimento); quer dizer, o conhecimento do que é adequado". Para Bentham, o objetivo da deontologia é a busca da felicidade, comparada ao "gozo do prazer isento de punição". Na França, o termo foi usado pela primeira vez, fora do âmbito da filosofia moral, em 1845, por M. Simon ${ }^{(3)}$. Contudo, o sentido da palavra evoluiu, uma vez que ele passou a ser referido tão somente à moral profissional. A reflexão deontológica se desenvolveu, ao longo do século XIX e no começo do século XX, essencialmente nas áreas da medicina e do direito (ao passo que, no caso do direito, o termo usado era "usos", rebatizado de "deontologia" somente a partir dos anos 1970).

Na medicina, as questões relacionadas a uma regulação deontológica são bastante claras: em primeiro lugar, está a defesa dos interesses dos pacientes contra certos profissionais sem escrúpulos (tanto no que concerne à qualidade dos cuidados, como no valor dos honorários); depois, mais genericamente, tem-se a reflexão sobre assuntos práticos que se colocam no exercício cotidiano da profissão (notadamente o segredo profissional ou a observância das prescrições); e, por fim, considerando-se uma prática que se desenvolve em um contexto econômico liberal, trata-se de propor regras de concorrência profissional (principalmente relativas à publicidade).

Ora, o conjunto desses objetivos é difícil de se alcançar sem uma organização que englobe os profissionais. Essa é a razão de a deontologia ser indissociável da ordem dos médicos: os médicos necessitam de uma associação profissional para aplicar a deontologia, a qual se constitui em um argumento político para a criação da própria instituição.

(2) BENTHAM, Jeremy. Déontologie ou science de la morale. Paris: Charpentier, 1834

(3) SIMON, Maxime. Déontologie médicale ou des droits et des devoirs des médecins dans l'état actuel de la civilisation. Paris: Baillière, 1845. 
O alcance prático da deontologia depende em grande parte de sua forma jurídica, cuja análise constitui a primeira etapa deste comentário crítico (I). Com o esclarecimento desse ponto, passa-se à exposição das questões tratadas pela deontologia (II). Em seguida, colocam-se em perspectiva os valores e as concepções profissionais dos quais a deontologia é o receptáculo, cerne deste comentário (III).

\section{AS FORMAS JURÍDICAS SUCESSIVAS}

A deontologia médica assumiu diferentes formas jurídicas, desde ser uma criação voluntária até tornar-se um decreto do Conselho de Estado (1). Com a integração da deontologia ao direito de Estado e suas implicações, essa conformação produziu várias consequências sobre as quais vale a pena se debruçar (2).

\section{Da criação voluntária ao decreto do Conselho de Estado}

Paralelamente ao fato de ter sido um objeto de discussão entre os profissionais de 1845 a 1940, a deontologia médica incorporou-se, a partir dos anos 1880 , aos códigos elaborados pelos sindicatos e associações profissionais. Assim, em 1899, a Associação dos Médicos de Gironde publicou os "conselhos profissionais e princípios de deontologia". De forma análoga, dois projetos de códigos foram adotados pela Associação National de Medicina, e o de 1929 teve votação unânime. Enfim, o principal sindicato médico da época, a Confederação dos Sindicatos Médicos Franceses (CSMF), publicou, em 1936, seu "regulamento de deontologia médica". Esta confederação havia organizado os "conselhos de família", instâncias disciplinares responsáveis pelo cumprimento das regras da profissão. Ao mesmo tempo, a partir do início do século XX, os médicos legistas, nas universidades, implantaram uma disciplina de deontologia. Assim, na virada do século XX, a deontologia, conjunto de direitos e deveres ensinado pelos médicos legistas, passou progressivamente de uma acepção moral a uma acepção jurídica, porém, unicamente nas regras internas das instituições profissionais, associações ou sindicatos. Bem, o alcance dessas produções normativas tinha um limite importante: a não adesão às instituições que as promoviam.

Assim, as principais organizações profissionais médicas trabalharam para que a deontologia fosse reconhecida pelo Estado. Um certo número de projetos de lei foram enviados ao Parlamento entre 1918 e 1938, com o objetivo de conseguir a organização de uma ordem profissional, particularmente encarregada de aplicar a deontologia. Porém, tanto em razão da instabilidade governamental como pelas particularidades do processo parlamentar da terceira República, nenhum destes projetos seguiu adiante. As ordens profissionais da área da saúde foram criadas durante a Segunda Grande Guerra Mundial, pelo 
governo de Vichy, que, de fato, foi adquirindo uma organização "corporativista" de economia e empregos, sobretudo, a fim de "vencer a luta de classes". A ordem dos médicos foi assim rapidamente criada após a instalação do regime de Vichy, em outubro de 1940. Sendo as ordens profissionais habilitadas a organizar seu funcionamento interno, a dos médicos adotou um código deontológico como seu regulamento interno, inspirado na deontologia elaborada em um contexto associativo e sindical, principalmente o da Confederação dos Sindicatos Médicos Franceses (CSMF).

A mudança de alcance jurídico foi radical: a ordem profissional obrigatoriamente reunia o conjunto dos profissionais. Suas regras e regulamentos internos se aplicavam a todos os profissionais, sem possibilidade de fuga. A deontologia se tornou então uma das normas do exercício da profissão médica, à qual cada profissional deveria se submeter.

Esse arranjo duraria muito tempo. De fato, quando, em 1944, o governo provisório (nascido da Resistência) anulou o conjunto de leis aprovados pelo regime de Vichy, definiram-se certas exceções, entre as quais as regras organizadoras das ordens profissionais. Porém, do ponto de vista do novo governo, não se tratou de promover a "superação da luta de classes" defendida por Vichy (os médicos raramente pertenciam ao proletariado), mas sim, de organizar a polícia administrativa das profissões. As ordens profissionais sobreviveram à "Libertação", todavia, com alguns ajustes referentes à deontologia. A maneira com que o código deontológico seria elaborado e validado foi alterada: desde então - e até hoje - ele deve ter o caráter de um regulamento da administração pública, sendo, atualmente, originário de um decreto do Conselho de Estado, o que significa que se trata de um ato do Primeiro Ministro, o topo da hierarquia dos atos administativos. Quatro códigos deontológicos se sucederam desde 1945: em 1947, 1955, 1979 e 1995. Constataremos que, em relação aos princípios essenciais, as normas deontológicas mantiveram-se estáveis.

\section{As consequências da integração ao direito de Estado}

A elaboração do código de deontologia médica se insere em um procedimento particular. O Art. L. 4121-7 do Código da Saúde Pública ${ }^{(4)}$ determina que "um código deontológico, próprio a cada uma das profissões de médico, cirurgião-dentista e parteira, preparado pelo conselho nacional da ordem interessada, é promulgado sob a forma de um decreto do Conselho de Estado". Nesse ponto, é necessário compreender que o Primeiro Ministro não pode promulgar ou modificar um código por iniciativa própria (o código é elaborado pelo conselho nacional da ordem).; porém, inversamente, a ordem profissional não

(4) FRANÇA. Code de la santé publique. Disponível em: <http://www.legifrance.gouv.fr/affichCode. do?cidTexte=LEGITEXT000006072665\&dateTexte=20120703>. Acesso em: 2 jul. 2012. 
pode promulgar ou modificar sua deontologia sem um acordo com o Primeiro Ministro (o qual delega, na prática, seu poder de negociação ao ministro da Saúde). Trata-se de uma exceção notável à maneira pela qual são habitualmente criados os atos administrativos, o que permite atestar o poder do profissional sobre as regras que se aplicam a ele. De um ponto de vista formal, o código deontológico médico foi objeto, em 2004, de um decreto não codificado: ele foi integrado, desde então, à parte regulamentadora do Código de Saúde Pública, nos artigos R. 4127-1 a R. 4127-112.

Essa organização normativa produz consequências sobre o alcance jurídico do texto. Na hierarquia das normas francesas, os decretos do Conselho de Estado, atos do Primeiro Ministro, situam-se acima dos decretos e resoluções do ministro da Saúde. Tal organização significa que o ministro da Saúde não pode criar regras que derroguem o código de deontologia médica. Por outro lado, a lei remete sistematicamente à deontologia quando intervém no campo da aplicação desta última. Por exemplo, o Código de Saúde Pública prevê, em sua parte legislativa, que o diretor de um hospital público "exerça sua autoridade sobre o conjunto de sua equipe com relação ao respeito às regras deontológicas ou profissionais que se impõem aos profissionais de saúde". Dito de outra forma, a autoridade do diretor do hospital termina onde começa a deontologia. A ordem dos médicos dispõe de um poder de controle sobre os contratos celebrados pelos seus associados no exercício de sua profissão (os médicos devem comunicar à ordem o conjunto de seus contratos profissionais), com o objetivo de verificar a ausência de contrariedade nesses documentos em relação à deontologia. Portanto, se o conselho da ordem não pode anular um contrato civil, ele pode impor sanções disciplinárias ao profissional que violar a deontologia, inclusive em um contrato. O conselho nacional da ordem propôs, nesse contexto, o "contrato-tipo" que respeita a deontologia e protege o profissional de surpresas desagradáveis no que concerne aos seus contratos. Enfim, do ponto de vista da responsabilidade profissional, a análise jurisprudencial mostra que as regras deontológicas podem, segundo o direito comum, servir de referência aos juízes, principalmente os civis, por qualificarem eventuais faltas no exercício da profissão.

Depois de apresentar as diferentes formas jurídicas assumidas pela deontologia médica no direito francês, passa-se a tratar de seus conteúdos, seus objetos.

\section{OS OBJETOS}

A deontologia médica tem por objeto a regulação do conjunto das relações profissionais, as quais podem-se dividir em: (1) as relações entre os próprios profissionais e (2) as relações dos profissionais com terceiros. 


\section{Relações entre os próprios profissionais}

Os relacionamentos entre profissionais se subdividem em duas categorias: fraternal e concorrencial.

\subsection{A fraternidade}

Como todas as outras, a deontologia médica afirma a importância da fraternidade, essencialmente traduzida, no código deontológico por: "os médicos devem prestar assistência uns aos outros em momentos de adversidade" (Art. 56, R. 4127-56, do Código de Saúde Pública). Essa obrigação de irmandade implica consequências institucionais na medida em que "o médico em disputa com um colega deve buscar a conciliação, usando, se necessário, o conselho departamental da ordem”. Porém, esta obrigação não é a mais importante.

\subsection{A concorrência}

O ponto mais importante ligado às relações profissionais reside, há mais de um século e meio, na concorrência entre os médicos. Essa questão mostrou-se fundamental em 1945, na medida em que os sistemas de proteção social dos assalariados não eram, até então, obrigatórios e que o acesso aos cuidados não eram, correlativamente, financiados pela coletividade. Nesse contexto, os rendimentos dos médicos dependiam muito estreitamente de sua capacidade de conquistar clientes, e certos profissionais sem escrúpulos podiam utilizar de meios reprováveis pela profissão. Evidencia-se aqui uma riqueza de vocabulário para definir práticas anticoncorrenciais: "cumplicidade", "comissão", "desconto", "taxas mais baixas" e outras práticas de "concorrência desleal”. A lógica dessas regras é a seguinte: "a medicina não deve ser praticada como um comércio" (Art.19) e as práticas que levem a pensar o contrário são proibidas. Essa é a razão para que, no mesmo artigo, tenha se acrescentado que "são proibidos todos os procedimentos diretos ou indiretos de publicidade, notadamente toda sinalização que dê ao local uma aparência comercial”. A única informação pública é regulamentada de maneira precisa, principalmente no que se refere à placa e aos documentos profissionais.

No mesmo sentido, os anos 1960 viram tomar corpo um debate sobre a possibilidade, por parte dos médicos, de serem proprietários de equipamentos ou de estabelecimentos hospitalares, assim como a possibilidade de se associarem com outros profissionais. Evidentemente, acreditava-se que o médico proprietário de um estabelecimento hospitalar não favoreceria a atividade do hospital em detrimento do respeito à deontologia; a associação entre médicos pareceu contrária a uma certa visão de exercício "liberal", solitário, sendo essa modalidade de prática, em uma primeira instância, considerada como uma "cumplicidade" (ou uma violação à deontologia). Nessas duas hipóteses, a lei 
aceitou as evoluções legais e regulamentadoras, mas na exata medida na qual a deontologia pode ser aplicada. É por essa razão, por exemplo, que o Art. 95 do Código afirma que "o fato de um médico estar vinculado, dentro de sua prática profissional, por meio de um contrato ou um estatuto a outro médico, empresa ou entidade pública ou privada não diminui seus deveres profissionais e, em particular, suas obrigações referentes ao sigilo profissional e à independência das suas decisões".

Pode-se destacar, considerando a importância prática da questão, que as sanções disciplinares impostas pelo Conselho Nacional da Ordem dos Médicos dizem respeito (cerca de $25 \%$ delas) a comportamentos relacionados à concorrência.

\section{Relações com terceiros}

Os códigos de deontologia foram, por muito tempo, apresentados como um conjunto de regras "de uso interno", um "direito fechado", tendo por origem os profissionais e tratando de questões que dizem respeito apenas a eles. Com esse argumento, a regulação deontológica não pode sofrer nenhuma crítica em termos de legitimidade.

Porém, o código deontológico médico, que reúne a quase totalidade das normas relativas ao exercício da profissão, trata também das relações entre os médicos e os não médicos, como os pacientes e seus funcionários.

\subsection{Relações com os pacientes}

A proteção dos pacientes é o argumento principal apresentado pelos médicos em sua reivindicação de uma regulação deontológica: a necessidade, para o bem dos pacientes, de que os profissionais obedeçam a regras que tratam de suas práticas cotidianas, com o objetivo de garantir a qualidade dos cuidados. Nesse contexto, compreende-se que o código deontológico retome, em seu Art. 40, o princípio tradicional primum non nocere, ao afirmar que "o médico, em suas investigações, intervenções ou terapêuticas, deve evitar que o paciente corra riscos desnecessários", e, no mesmo sentido, o Art. 39 prevê que "os médicos não podem propor, aos doentes ou aos seus familiares, um medicamento ou procedimento ilusório ou não totalmente testado, como saudável ou inofensivo. Toda prática de charlatanismo é proibida". De maneira mais abrangente, o Art. 2 do código prevê que "o médico, na prestação de serviços às pessoas ou à saúde pública, exerce sua missão dentro do respeito à vida humana, à pessoa e a sua dignidade. O respeito devido à pessoa não cessa de existir após a morte".

Certos princípios referentes às relações entre os médicos e seus pacientes foram debatidos em 2002, quando da discussão da Lei $n^{\circ} 2002-303$, que trata dos direitos dos pacientes. O Art. 35 alínea 2 do Código de Deontologia Médica previa, até aquela data, que o médico podia não informar o paciente 
"em caso de prognóstico ou diagnóstico grave", situação sobre a qual ele "refletiria com consciência". Tal construção significava que a decisão de informar ou não o paciente seria do médico, sob o controle único de sua consciência, regra inconciliável com o direito do paciente de ser informado (exceto se ele não o desejar), reivindicação das associações de pacientes nos anos 1990. A lei consagrou o direito do paciente à informação e, com relação a esse ponto, o código deontológico foi tacitamente anulado. Mas tal debate permitiu mostrar que, se o código de deontologia foi construído para defender o interesse dos pacientes, trata-se dos interesses dos pacientes dentro da concepção dos médicos, e não da dos doentes; o exemplo apresentado permite constatar que os conceitos sobre o tema podem divergir.

\subsection{Relações com os empregados}

Uma das questões marcantes da história da construção da deontologia médica refere-se à independência dos profissionais. Desde os anos 1920 que o princípio da independência vem sendo afirmado, com o objetivo de escapar a uma eventual influência dos empregadores sobre as práticas médicas. Essa é a razão do Art. 5, segundo o qual "o médico não pode alienar sua independência profissional em nenhuma situação". Em relação ao exercício assalariado da medicina, o Art. 95 do código confirma o princípio da independência ao prever que "um médico ligado em sua prática profissional, por contrato ou estatuto, a outro médico, a uma administração, coletividade ou instituição pública ou privada, não diminui seus deveres profissionais e, em particular, suas obrigações em matéria de confidencialidade e independência de suas decisões"; em sua alínea segunda, o artigo determina que, em relação ao assalariado, "em nenhuma circunstância, o médico pode aceitar limitações a sua independência em sua prática profissional da parte de outro médico, empresa ou instituição que o empregue. Ele deve sempre agir, prioritariamente, no interesse da saúde púbica e no interesse das pessoas e de sua segurança, dentro das empresas ou instituições em que atua". No mesmo sentido, o Art. 97 acrescenta que "um médico assalariado não pode, em nenhum caso, aeitar remuneração com base em normas de produtividade, rendimento por hora ou qualquer dispositivo que tenha por consequência uma limitação ou abandono de sua independência ou que afete a qualidade dos cuidados médicos". Deve-se entender que, qualquer que seja o cenário jurídico no qual o médico atue, aplica-se o princípio da independência relativo às decisões médicas. Pode-se, entretanto, retrucar que o que se diz na deontologia não encontra necessariamente aplicação prática, pelo seu alcance limitado, afirmado apenas em um decreto do Conselho de Estado. Porém, vale lembrar, como dito em relação às consequências da integração da deontologia ao direito do Estado, que as leis que interveem no domínio da deontologia referem-se sistematicamente a ela quando se está em seu campo de aplicação.

Pelo que foi explicitado até o momento, compreende-se que nem a maneira com que as regras deontológicas são elaboradas, nem seus objetos estão 
livres de crítica. Passa-se agora ao desenvolvimento da ideia que busca mostrar que a deontologia constitui de fato um instrumento de defesa das concepções "tradicionais" da medicina.

\section{A DEONTOLOGIA, INSTRUMENTO DE DEFESA DE CONCEPÇÕES TRADICIONAIS DA MEDICINA?}

De acordo com o Código de Saúde Pública (Art. L. 4121-1), a ordem dos médicos "reúne obrigatoriamente todos os médicos (...) habilitados a exercer a medicina". Nesse contexto, a ordem é responsável por "velar pela manutenção dos princípios de moralidade, probidade, competência e devotamento indispensáveis ao exercício da medicina (...) e à observação, por parte de todos os seus integrantes, dos deveres profissionais, assim como das regras do Código de Deontologia previsto pelo Art. L. 4127-1", acrescentando que ele "garante a defesa da honra e a independência da profissão médica". O conjunto desses enunciados referentes à manutenção, observação dos deveres e regras da deontologia e também à defesa da honra e da independência remete à defesa de certos valores e de concepção da medicina que se pode qualificar de tradicionais, na medida em que eles figuram na lei desde 1945, atendendo a uma reivindicação expressa pelos médicos desde 1845. Essa é uma das críticas recorrentes que se faz à ordem dos médicos (principalmente com relação à resistência a certas evoluções sociais, como a contracepção nos anos 1960 ou a interrupção da gravidez nos anos 1970): ela seria conservadora, quiçá reacionária. Se o segundo epíteto pode ser discutido, o primeiro não surpreende o meio jurídico, uma vez que a ordem profissional é justamente concebida para agir de maneira conservadora.

Compreende-se então que a deontologia, elaborada pela ordem que deve garantir o respeito às regras, se inscreve em uma mesma lógica.

Passa-se agora à discussão de três questões: a defesa da medicina liberal (1); o paternalismo médico (2) ; e a independência profissional (3).

\section{A medicina liberal}

Como exposto anteriormente, as regras de quatro códigos deontológicos que se sucederam desde 1947 se fundiram nos princípios do regulamento interno do Conselho Nacional da Ordem dos Médicos de 1941, que retoma, ele mesmo, os princípios da "carta médica" de 1927. Ora, é importante conhecer as questões envolvidas nessa produção, as quais deixaram um traço durável no Código de Deontologia (e no conjunto do direito francês). O contexto no qual a carta foi redigida foi de fortalecimento da medicina social e de preparação de uma lei sobre as sociedades de seguro social, as organizações de seguridade social 
responsáveis pelos custos da prestação de serviço. Um sindicato minoritário de médicos, opositores da medicina social, elaborou uma carta e conseguiu, após alguns périplos políticos, aprovar os princípios do documento junto ao governo. A carta da medicina liberal apresentava cinco princípios referentes a questões deontológicas: a liberdade do paciente para escolher o médico; o segredo profissional; o direito de receber honorários por cada paciente tratado; o "acordo direto" entre o profissional e o paciente, pelo qual o paciente paga diretamente ao médico, sem a interferência de um terceiro (o paciente pode ser reembolsado depois); a liberdade terapêutica (da qual temos a liberdade de prescrição). Percebe-se que, na época, tais princípios impediam - e era essa sua meta - a possibilidade de organizar o exercício da medicina assalariada (principalmente pelo pagamento direto e no fechamento do contrato). Seria necessário esperar mais de 30 anos e uma lei para que a medicina assalariada se tornasse possível. Porém, ainda hoje, segundo o Art. L. 162-2, do Código de Seguridade Social - que trata dos princípios fundamentais que governam as relações entre os médicos e o seguro saúde (seguro obrigatório para tratamentos) --, "no interesse de garantias sociais e da saúde pública, no respeito à liberdade do exercício e da independência profissional e moral dos médicos é assegurado, conforme os princípios deontológicos fundamentais: a livre escoIha dos médicos por parte dos pacientes, a liberdade de prescrição médica, o segredo profissional, o pagamento direto de honorários por parte do paciente e a liberdade de estabelecimento do médico".

A deontologia constitui-se como padrão de defesa - contendo todos os princípios - de uma certa concepção de medicina liberal. Destaque-se que certos princípios estão hoje bastante modificados principalmente pelo direito de seguro saúde. Nessa hipótese, as regras deontológicas são aplicáveis; porém, na medida em que mais de $99 \%$ dos médicos são conveniados (as regras referentes às relações entre o seguro saúde e os médicos foram objeto de "convenções" entre as instituições de seguro saúde e os sindicatos profissionais), a exceção torna-se, estatisticamente, a regra. É por essa razão, por exemplo, que se desenvolveu, principalmente entre os pacientes pobres, o sistema do "terceiro pagante", que constitui uma derrogação do princípio de "acordo direto".

\section{O paternalismo médico}

A doutrina dita do "paternalismo médico" foi teorizada nos anos 1950, pelo presidente do Conselho Nacional da Ordem dos Médicos, Louis Portes, que escreveu: "em face do paciente, inerte e passivo, o médico não tem, de modo algum, o sentimento de lidar com um sujeito livre, um igual, um par, que ele possa verdadeiramente instruir. Todo paciente é, e deve ser para ele, como uma criança a ser domada, certamente não para enganar - uma criança para consolar, não abusar - uma criança para salvar ou simplesmente para curar, por meio das vicissitudes do desconhecido". Essa doutrina deu aos médicos 
um verdadeiro "direito de curar"; o paciente devia se contentar em obedecer ao médico para assegurar sua cura. Uma questão ilustrativa dessa concepção é a observância da prescrição médica. Dessa maneira, o Código de Deontologia Médica de 27 de junho de 1947 previa, em seu Art. 30, que, "após ter-se estabelecido um diagnóstico definitivo que implica uma decisão séria, sobretudo se a vida do doente está em perigo, um médico deve se esforçar para impor a execução de sua decisão" (grifo nosso). O Código de 28 de novembro de 1955, no Art. 29, definia que o profissional devia "se esforçar para conseguir a execução do tratamento prescrito". Já o Código de 28 de junho de 1979, no Art. 37, afirmava que o médico devia "se esforçar para obter uma boa execução do tratamento". Por fim, o Código de 6 de setembro de 1995 previa que "O médico deve formular suas prescrições com toda a clareza indispensável, cuidando que elas sejam compreendidas pelo paciente e sua família, e se esforçar para conseguir uma boa execução". Constata-se que, se as formulações evoluíram e perderam seu vigor, a lógica subjacente continuou a mesma: o paciente deve obedecer a prescrição.

Essa concepção "imperialista" do exercício médico - mesmo que progressivamente tenha sido alterada até os anos 2000 -, negando ao paciente a capacidade de decidir, levou a profissão médica - ou ao menos sua ordem profissional - a se opor, em 2002, à evolução das regras relacionadas ao direito do paciente à informação. Até a Lei $n^{\circ} 2002-303$, de 4 de março de 2002, de fato, a ordem dos médicos sempre foi a responsável pelas regras referentes ao direito do paciente à informação, que estão definidas no Art. 35 do Código de Deontologia, cuja alteração, segundo o Art. L. 4121-7 do Códigio de Saúde Pública, também é prerrogativa da ordem dos médicos. Poderíamos imaginar que o Código de Deontologia Médica evolui, por iniciativa da ordem dos médicos, para corresponder à evolução das normas sociais, como atestam as regras decorrentes da lei sobre os direitos dos pacientes, caso em que as mudanças foram tecnicamente inúteis. Na falta de tal iniciativa, a ordem dos médicos levou o legislador a intervir em um domínio relevante e tradicional da regulação deontológica. A regulamentação dos direitos do pacientes à informação escapa da regulação deontológica, uma vez que o Art. L. 1111-2, alínea 6, do Código de Saúde Pública, prevê que "as recomendações de boas práticas sobre o fornecimento de informação são estabelecidas pela Agência Nacional de Acreditação e Avaliação em Saúde e homologadas por decreto do ministro da Saúde". Antes da lei de 4 de março de 2002, os médicos tinham deontologicamente o direito de informar ou não seus pacientes em caso de prognóstico ou diagnóstico graves, sem prestar contas a não ser a sua própria consciência; depois de 4 de março de 2002, eles têm o dever de informar seus pacientes, que, desde então, dispõem de um verdadeiro direito subjetivo de serem informados. A deontologia, por muito tempo receptáculo de regras relativas ao direito do paciente à informação, deve hoje se contentar em seguir a lei, determinada pelas recomendações de boas práticas. 
Uma lição mais geral pode ser tirada: desde 2002, a deontologia não engloba mais o conjunto do comportamento dos profissionais. Outras questões também deontológicas foram objeto de uma intervenção legal depois de 2002: a definição do fim da vida (2005), informação sobre honorários (2007) e a discriminação contra pacientes pobres (2010).

\section{A independência profissional}

Um dos fundamentos tradicionais da deontologia reside na independência do profissional. A ideia básica é que, por estar a serviço do paciente, o profissional deve ser independente de qualquer interferência. A orientação efetiva da atividade pelo interesse do paciente remete à consciência do profissional; uma fórmula tradicional para resumir a relação médica é: "o encontro entre a consciência e a confiança".O princípio da independência profissional está inscrito na abertura do código deontológico, em um de seus primeiros artigos, o Art. 5, segundo o qual "o médico não pode alienar sua independência profissional de nenhuma maneira" e também faz parte de outros artigos, como o Art. 95, relativo ao médico assalariado, que afirma que "o fato de um médico estar vinculado, dentro de sua prática profissional, por meio de um contrato ou um estatuto a outro médico, empresa ou entidade pública ou privada não diminui seus deveres profissionais e, em particular, suas obrigações referentes ao sigilo profissional e à independência das suas decisões”. Essa exigência é retransmitida, para além do código deontológico, por lei, principalmente para o Código de Saúde Pública que, a propósito dos médicos agentes públicos, afirma que "o diretor (de um hospital) exerce sua autoridade sobre o conjunto das pessoas dentro do respeito às regras deontológicas ou profissionais (...) e do respeito à independência profissional do médico no exercício de seu ofício”.

Certas questões deontológicas tradicionais, que não têm por objeto explícito a independência, podem ser analisadas como defesas indiretas desse princípio. Esse é o caso do segredo profissional. O discurso dos profissionais a esse propósito é bastante firme, até mesmo retomando, para o legitimar, o princípio do segredo de Hipócrates (esquecendo, de passagem, um eclipse de cerca de dois mil anos de história que não reconhecia este princípio do segredo profissional, indo desde o Império Romano até o século XVIII...). Na França, o segredo profissional, incluído no Código Penal em 1810, tem como objetivo a proteção da vida privada das pessoas; ele é retomado, depois do século XIX, nas diferentes deontologias. Mas, se para os pacientes seu interesse é inegável, o segredo constitui igualmente uma garantia da independência para os profissionais, permitindo que eles escapem de demandas de informações relativas a suas atividades e que eles fiquem fora do alcance de controles externos.

Porém, paralelamente, os médicos reivindicam constantemente a possibilidade de não serem submetidos ao princípio do segredo profissional, desde 
que a discussão seja entre eles. Há fortes presunções de que eles de fato, mas de maneira ilegal, não se submetiam a este princípio antes da lei de 4 de março, uma vez que eles acreditavam estarem tomando essa atitude em defesa do interesse do paciente (ou de sua própria concepção do que seria este interesse...). Tratava-se, em todo caso, de uma forte reivindicação profissional que, a partir de 2002, foi atendida com o Art. L. 1110-4, do Código de Saúde Pública, que afirma que "toda pessoa em tratamento (...) tem o direito ao respeito a sua vida privada e ao segredo das informações que lhe são concernentes". Contudo, o mesmo dispositivo prevê que "dois ou mais profissionais de saúde podem, salvo oposição do paciente devidamente informado, trocar informações relativas a uma mesma pessoa em tratamento, a fim de assegurar a continuidade dos cuidados ou de determinar o melhor tratamento de saúde possível".

Em face das regras do Código Penal que visam limitar a independência dos médicos ao impor-lhes o segredo profissional, pode-se constatar que eles reivindicaram, e conseguiram, uma derrogação desse princípio geral, respeitando sua independência e englobando quase a totalidade das discussões profissionais. Os médicos podem trocar informações sobre um paciente com outros médicos, no exercício de sua profissão (a fim de assegurar a continuidade do tratamento ou determinar a melhora dos cuidados...), o que corresponde a sua reivindicação. O segredo profissional continua sendo o princípio que trata das relações entre médicos e não médicos e pode-se afirmar que este princípio atende igualmente aos interesses dos pacientes ; porém, é preciso constatar que o sistema não frustra os anseios dos profissionais da saúde.

Com relação a essa discussão, pode-se objetar que se trata de um debate relativo às normas legais, o que é tecnicamente o caso; no entanto, deve-se entender que, para os profissionais e para os parlamentares, a questão é principalmente deontológica e não constitui uma questão legal, a não ser secundariamente.

Aqui, outros pontos poderiam ser analisados: a definição do fim da vida, a administração das discriminações contra pacientes pobres, a fixação dos honorários etc. Destaque-se ainda que a mesma lógica da independência esteve presente nas reflexões sobre o exercício liberal da medicina e sobre o paternalismo médico. A defesa da independência profissional constitui-se assim como um princípio deontológico fundamental e pode ser mesmo o princípio deontológico básico, influenciando o conjunto da deontologia.

\section{CONSIDERAÇÕES FINAIS}

O Código de Deontologia Médica constitui uma norma fundamental para o exercício da medicina, sendo, até recentemente, o instrumento exclusivo para o ordenamento do conjunto de regras relativas às relações profissionais dos 
médicos. O código poderia ser considerado um verdadeiro "código de exercício profissional". Hoje, essa afirmação deve ser relativizada: desde a lei de 4 de março de 2002, que trata dos direitos dos pacientes, uma parte das regras referentes às relações entre médicos e pacientes passou a ter como base o Código de Saúde Pública. Desde 2002, a deontologia deixou de englobar o conjunto do comportamento profissional. Dessa maneira, a lei de 4 de março de 2002 mostrou um inegável avanço democrático, pondo fim a uma hipocrisia institucional: a elaboração dos códigos deontológicos pelos profissionais, com base na crença de que as regras dizem respeito apenas a esses profissionais, enquanto, na verdade, várias delas que envolvem outras partes - em particular relacionadas ao direito à informação ou ao consentimento dos pacientes ou ainda à independência profissional e, indiretamente, ao poder dos empregadores - figuravam ou figuram no código deontológico.

O conteúdo do Código de Deontologia Médica pode ser apresentado como referência a duas funções por ele asseguradas: de um lado, instrumento de controle da profissão médica e, de outro, instrumento de independência profissional vis-à-vis em relação a outros interlocutores.

Por meio do código deontológico, a ordem dos profissionais médicos pode fiscalizar contratos quanto a sua adequação às normas deontológicas, bem como sancionar disciplinarmente um profissional antiético. A independência profissional é assegurada pelas disposições do código deontológico que a reconhece, à qual a lei remete sistematicamente e que constitui a norma de referência relativa às interações do médico com o conjunto de seus interlocutores.

O código deontológico é, enfim, uma construção técnica que remete a valores e concepções da profissão médica que podem ser impostos aos profissionais recalcitrantes e aos não profissionais. Foi necessário esperar até 2002 para que o legislador o contrariasse no que concerne ao tratamento dos direitos do paciente à informação. Se os médicos tivessem optado por alinhar as disposições do código às concepções sociais dos pacientes e não as suas, as regras estariam ainda no código deontológico. Mas, eles não escolheram esse caminho.

\section{REFERÊNCIAS}

ALMERAS, Jean-Pierre; PÉQUIGNOT, Henri. La déontologie médicale. Paris: Litec, 1996.

BENTHAM, Jeremy. Déontologie ou science de la morale. Paris: Charpentier, 1834. $2 \mathrm{v}$.

FRANÇA. Code de déontologie médicale. Disponível em : <http://www.conseilnational.medecin.fr/groupe/17/tous>. Acesso em: 2 jul. 2012. 
FRANÇA. Code de la santé publique. Disponível em: <http://www.legifrance. gouv.fr/affichCode.do?cidTexte=LEGITEXT000006072665\&dateTex te $=20120703>$. Acesso em: 2 jul. 2012.

HATZFELD, Henri. Le grand tournant de la médecine libérale. Paris: Éditions Ouvrières, 1963.

JAUNAIT, Alexandre. Comment pense l'institution médicale? Paris: Dalloz, 2005. (Nouvelle Bibliothèque de Thèses).

LEONARD, Jacques. La médecine entre les pouvoirs et les savoirs: histoire intellectuelle et politique de la médecine au XIXe siècle. Paris: AubierMontaigne, 1981.

MORET-BAILLY, Joël. Les déontologies. Aix-en-Provence: Presses Universitaires d'Aix-Marseille, 2001.

ORDRE NATIONAL DES MEDECINS. Commentaire du code de déontologie. Disponível em: <http://www.conseil-national.medecin.fr/groupe/17/tous>. Acesso em: 22 maio 2012.

PORTES, Louis. A la recherche d'une éthique médicale. Paris: Masson, 1954.

RODWIN, Marc. Conflicts of interest and the future of medicine. Oxford: Oxford University Press, 2011.

SIMON, Maxime. Déontologie médicale ou des droits et des devoirs des médecins dans l'état actuel de la civilisation. Paris: Baillière, 1845.

THOUVENIN, Dominique. Le secret médical et l'information du malade. Lyon: Presses Universitaires de Lyon, 1982. 


\section{VERSÃO ORIGINAL}

\section{LE CODE FRANÇAIS DE DÉONTOLOGIE MÉDICALE: COMMENTAIRE CRITIQUE}

Joël Moret-Bailly*

La réflexion relative à la déontologie médicale nait, en France, au milieu du XIXe siècle, s'inspirant de l'organisation professionnelle des professions de justice et du droit (avocats, notaires, huissiers, commissaires-priseurs...) datant des années 1810.

Dans ce contexte le terme "déontologie" reprend le mot inventé en 1834 par le philosophe utilitariste anglais Jeremy Bentham ${ }^{(1)}$. Le philosophe explique que "le mot Déontologie est dérivé de deux mots grecs, deon-ontos (ce qui est convenable) et logos (connaissance); c'est à dire, la connaissance de ce qui est juste et convenable". L'objectif que Bentham assigne à la déontologie est la recherche du bonheur, assimilé à "la possession du plaisir avec exemption de peine". Le terme est utilisé, en France, pour la première fois en dehors de la philosophie morale, en 1845 dans un ouvrage de $M$. Simon ${ }^{(2)}$. Son sens a cependant évolué puisqu'il ne concerne, et ne concernera plus, dès lors, que la morale professionnelle. La réflexion déontologique va alors se développer tout au long du XIXe et au début du XXe siècle, essentiellement dans les professions de médecin et d'avocat (mais, pour ces derniers, sous le vocable "d'usages", rebaptisés "déontologie" seulement à partir des années 1970).

En médecine, les enjeux liés à une régulation déontologique sont alors très clairs: il s'agit tout d'abord de défendre l'intérêt des patients contre certains professionnels indélicats (tant en ce qui concerne la qualité de la prise en charge que le montant des honoraires); il s'agit, plus généralement, de réfléchir à des questions pratiques se posant dans l'exercice quotidien de la profession (notamment le secret professionnel ou l'observance des prescriptions); il s'agit, enfin, dans une pratique qui se développe dans un cadre économique libéral, de proposer des règles de concurrence professionnelle (notamment les règles relatives aux plaques professionnelles ou à la publicité).

(*) Professeur de droit privé et sciences criminelles, Université de Lyon, Université Jean Monnet. Saint-Étienne/France.

(1) BENTHAM, Jeremy. Déontologie ou science de la morale, revu, mis en ordre et publié par John Bowring, traduit sur le manuscrit par Benjamin Laroche, deux tomes, Paris: Charpentier, 1834.

(2) SIMON, Maxime. Déontologie médicale ou des droits et des devoirs des médecins dans l'état actuel de la civilisation. Paris: Baillière, 1845. 
Or, l'ensemble de ces objectifs s'avère difficile à atteindre sans une organisation encadrant l'ensemble de ceux qui exercent la profession. C'est la raison pour laquelle l'histoire de la déontologie est indissociable de celle de l'ordre des médecins: les médecins ont besoin d'un ordre professionnel pour faire appliquer la déontologie; la déontologie constitue un argument politique pour la création de l'ordre.

La portée pratique de la déontologie dépendant grandement de sa forme juridique, l'analyse de cette dernière constitue la première étape d'un commentaire critique de la déontologie (I). Une fois cette question clarifiée, il devient loisible d'exposer les questions traitées, au fond, par la déontologie (II). Ce second élément éclairé, il est possible de mettre en perspective les valeurs et les conceptions professionnelles dont la déontologie constitue le réceptacle - ainsi que le fer de lance -, ce dernier élément constituant le cœur de son commentaire critique (III).

\section{LES FORMES JURIDIQUES SUCCESSIVES}

La déontologie médicale a successivement emprunté plusieurs formes juridiques, de l'élaboration volontaire au décret en Conseil d'État (1), cette dernière forme, du fait de l'intégration de la déontologie au droit de l'État qu'elle implique, produisant nombre de conséquences sur lesquelles il faut insister (2).

\section{De l'élaboration volontaire au décret en Conseil d'Etat}

Parallèlement au fait de constituer un objet de débats entre professionnels de 1845 à 1940, la déontologie médicale va s'incarner, à partir des années 1880, dans des codes élaborés par des syndicats et des associations professionnelles. Ainsi en 1899, l'Association des médecins de la Gironde publie des "conseils professionnels et principes de déontologie". Parallèlement, deux projets de codes sont adoptés par l'Académie nationale de médecine, dont l'un, en 1929, à l'unanimité. Enfin, le principal syndicat médical de l'époque, la Confédération des syndicats médicaux français (CSMF), publie, en 1936, son "règlement de déontologie médicale". Cette confédération avait même organisé des "conseils de famille", instances disciplinaires chargées de faire respecter les règles de la profession. Parallèlement se développe même, à partir du début du XXe siècle, un enseignement de déontologie à l'Université par des médecins légistes. Ainsi, au tournant du XXe siècle, la déontologie, ensemble de droits et de devoirs, enseignée par des médecins-légistes, passe progressivement d'une acception morale à une acception juridique, mais uniquement dans les règles internes d'institutions professionnelles, associations ou syndicats. Or, la portée de ces productions normatives connaît une limite importante: il suffit, pour leur échapper, de ne pas adhérer aux institutions qui les promeuvent. 
Les principales organisations professionnelles médicales vont donc œuvrer à faire reconnaître la déontologie par l'État. Un certain nombre de projets de loi sont notamment déposés au Parlement entre 1918 et 1938, dans le but d'obtenir l'organisation d'un ordre professionnel, notamment chargé d'appliquer la déontologie. Mais, du fait tant de l'instabilité gouvernementale que des particularités de la procédure parlementaire de la troisième République, aucun de ces projets ne verra le jour. Les ordres professionnels des professions de santé seront donc créés pendant la seconde guerre mondiale, par le régime de Vichy (hors le monde de la justice, organisé, quant à lui, rappelons le, au début du XIXe siècle). Le régime de Vichy est en effet acquis à une organisation "corporatiste" de l'économie et des métiers, notamment dans le but de "dépasser la lutte des classes". L'ordre des médecins est ainsi très rapidement créé après l'installation du régime de Vichy, en octobre 1940. Les ordres professionnels ayant compétence pour organiser leur fonctionnement interne, l'ordre des médecins adoptera alors un code de déontologie sous couvert de son règlement intérieur, qui s'inspire très largement de la déontologie élaborée dans un cadre associatif et syndical, notamment celle de la Confédération des syndicats médicaux français (CSMF).

Le changement de portée juridique est alors radical: l'ordre professionnel regroupe, en effet, obligatoirement l'ensemble des professionnels. Dès lors, ses règles, et notamment son règlement intérieur, s'appliquent à tous les professionnels, sans possibilité d'y échapper régulièrement. La déontologie devient donc l'une des normes de l'exercice de la profession médicale, et chaque professionnel lui est soumis.

Et cette organisation va perdurer. En effet, lorsqu'en 1944, le Gouvernement provisoire (issu de la Résistance) annule l'œuvre législative du régime de Vichy, il prévoit quelques exceptions à cette annulation générale, dont font partie les règles qui organisent les ordres professionnels. Mais il ne s'agit pas, de son point de vue, de permettre le "dépassement de la lutte des classes" prôné par Vichy (les médecins appartiennent rarement au prolétariat), mais seulement d'organiser la police administrative des professions. Les ordres professionnels vont donc survivre à la Libération, mais avec un certain nombre d'ajustements en ce qui concerne la déontologie. Son édiction est, en effet, réorganisée: elle prendra dorénavant - et jusqu'à aujourd'hui - la forme d'un règlement d'administration publique, aujourd'hui un décret en Conseil d'État, c'est à dire un acte du Premier ministre, au sommet de la hiérarchie des actes administratifs. Quatre codes de déontologie se sont ainsi succédés depuis 1945, en 1947, 1955, 1979 et 1995; nous constaterons, cependant, que sur leurs principes essentiels, les règles déontologiques sont restées tout à fait stables.

\section{Les conséquences de l'intégration au droit de l'État}

L'élaboration du code de déontologie médicale s'inscrit cependant dans une procédure particulière. L'article L. 4121-7 du code de la santé publique (pour 
utiliser la numérotation actuelle) prévoit en effet qu' 'un code de déontologie, propre à chacune des professions de médecin, chirurgien-dentiste et sage-femme, préparé par le conseil national de l'ordre intéressé, est édicté sous la forme d'un décret en Conseil d'Etat'. II faut ici comprendre que le premier ministre ne peut pas édicter ou modifier le code de déontologie de sa propre initiative (celui-ci est préparé par le conseil national de l'ordre); mais inversement, l'ordre professionnel ne peut édicter ou modifier sa déontologie et sans accord de premier ministre (ce dernier déléguant, en pratique, son pouvoir de négociation au ministre de la santé). II s'agit ici d'une exception notable à la manière dont sont habituellement produits les actes administratifs, qui permet d'attester le pouvoir de la profession sur les règles qui s'appliquent à elle. D'un point de vue formel, le code de déontologie médicale faisait l'objet, jusqu'en 2004, d'un décret non codifié; il est intégré, depuis cette date, à la partie réglementaire du code de la santé publique, aux articles R. 4127-1 à R. 4127-112.

Cette organisation normative produit, en outre, des conséquences sur la portée juridique du texte. Dans la hiérarchie des normes françaises, en effet, les décrets en Conseil d'État, actes du premier ministre, se situent au-dessus des décrets et arrêtés émanant du ministre de la santé. Une telle organisation signifie que le ministre de la santé ne peut régulièrement pas prendre de règles qui dérogeraient au code de déontologie médicale. La loi renvoie, en outre, elle-même systématiquement à la déontologie dès lors qu'elle intervient dans le champ d'application de cette dernière. Par exemple, le code de la santé publique prévoit, dans sa partie législative, que le directeur d'un hôpital public "exerce son autorité sur l'ensemble du personnel dans le respect des règles déontologiques ou professionnelles qui s'imposent aux professions de santé". Autrement dit, l'autorité du directeur de l'hôpital s'arrête là où commence la déontologie. L'ordre des médecins dispose, enfin, d'un pouvoir de contrôle sur les contrats passés par les médecins dans l'exercice de leur profession (ceux-ci devant communiquer à l'ordre l'ensemble de leurs contrats professionnels), dans le but de vérifier l'absence de contrariété de ces derniers à la déontologie. Dès lors, si le conseil de l'ordre ne peut annuler un contrat civil, il peut disciplinairement sanctionner le professionnel qui violerait la déontologie, y compris dans un contrat. Le conseil national de l'ordre propose, dans ce contexte, des "contrats-type" qui respectent la déontologie et mettent le professionnel à l'abri d'une mauvaise surprise en ce qui concerne ses contrats professionnels. Enfin, du point de vue de la responsabilité professionnelle, l'analyse jurisprudentielle montre que les règles déontologiques peuvent, conformément au droit commun, servir de référence aux juges, notamment civils, pour qualifier d'éventuelles fautes dans l'exercice de la profession.

Nous pouvons à présent, après avoir envisagé les différentes formes juridiques empruntées par la déontologie médicale en droit français, aborder la question de ses contenus, de ses objets. 


\section{LES OBJETS}

La déontologie médicale a, comme on l'a compris dans le I, pour objet la régulation de l'ensemble des relations professionnelles. On peut distribuer ces dernières en deux catégories: les rapports des professionnels entre eux (1), et les rapports entre les professionnels et les tiers (2).

\section{Rapports des professionnels entre eux}

Les rapports des professionnels entre eux peuvent se subdiviser en deux catégories: les rapports confraternels et les rapports concurrentiels.

\subsection{La confraternité}

La déontologie médicale affirme, comme toutes les autres déontologies, l'importance de la confraternité, essentiellement traduite, dans le code de déontologie, par la formule suivante: "les médecins se doivent assistance dans l'adversité" (article 56, soit R. 4127-56 du code de la santé publique). Cette obligation de confraternité entraîne des conséquences institutionnelles dans la mesure où "un médecin qui a un différend avec un confrère doit rechercher une conciliation, au besoin par l'intermédiaire du conseil départemental de l'ordre". Mais là n'est pas le plus important.

\section{2. La concurrence}

La question la plus importante en ce qui concerne les rapports des professionnels entre eux réside, depuis plus d'un siècle et demi, dans les rapports concurrentiels entre professionnels. Cette question était absolument fondamentale jusqu'en 1945 dans la mesure où les systèmes de protection sociale des salariés n'étaient, jusqu'à cette date, pas obligatoires et que l'accès aux soins n'était, corrélativement, pas financé par la collectivité. Dans ce contexte, les revenus des médecins dépendaient étroitement de leur capacité à attirer la clientèle, et certains professionnels indélicats pouvaient utiliser, pour ce faire, des moyens réprouvés par la profession. En témoigne la richesse du vocabulaire forgé à ce propos, les pratiques anticoncurrentielles se déclinant en "compérage", "commission", "ristourne", "abaissement d'honoraires", et autres pratiques de "concurrence déloyale". La logique de ces règles est la suivante: "la médecine ne doit pas être pratiquée comme un commerce" (article 19), et les pratiques qui pourraient donner à penser le contraire sont prohibées. C'est la raison pour laquelle le même article ajoute que "sont interdits tous procédés directs ou indirects de publicité et notamment tout aménagement ou signalisation donnant aux locaux une apparence commerciale". La seule information du public est alors 
règlementée de manière précise, notamment en ce qui concerne la plaque et les documents professionnels.

Dans le même registre, les années 1960 ont vu prendre place un débat sur la possibilité, pour les médecins, d'être propriétaire des équipements de soins ou des établissements hospitaliers, ainsi que de la possibilité, pour eux, de s'associer avec d'autres professionnels. La crainte était, évidemment, que le médecin propriétaire d'un établissement hospitalier ne favorise l'activité de l'établissement au détriment du respect de la déontologie; l'association entre médecins paraissait, quant à elle, contraire à une certaine vision de l'exercice "libéral" c'est-à-dire solitaire, cette modalité d'exercice étant même, dans un premier temps, considérée comme un "compérage" (c'est à dire une violation de la déontologie). Dans ces deux hypothèses, la loi a accepté des évolutions légales et réglementaires, mais dans la stricte mesure dans laquelle la déontologie trouverait toujours à s'appliquer. C'est à ce titre, par exemple, que selon l'article 95 du code, "le fait pour un médecin d'être lié dans son exercice professionnel par un contrat ou un statut à un autre médecin, une administration, une collectivité ou tout autre organisme public ou privé n'enlève rien à ses devoirs professionnels et en particulier à ses obligations concernant le secret professionnel et l'indépendance de ses décisions".

On peut souligner, enfin, et pour constater l'importance pratique de la question, que les sanctions disciplinaires prononcées par le conseil national de l'ordre des médecins concernent, pour près de $25 \%$, des comportements relatifs à la concurrence.

\section{Rapports avec les tiers}

Les codes de déontologie ont longtemps été présentés comme des ensembles de règles "à usage interne", un "droit clos", ayant pour origine les professionnels et ne concernant qu'eux. Il est vrai que l'argument est également souvent utilisé par les professionnels eux-mêmes, la régulation déontologique ne pouvant souffrir alors aucune critique en termes de légitimité.

Pourtant, le code de déontologie médicale, qui réunit la quasi-totalité des normes relatives à l'exercice de la profession, concerne également les rapports entre les médecins et les non médecins. Tel est notamment le cas en ce qui concerne les règles relatives aux rapports entre les professionnels et les patients ainsi que celles relatives à leurs rapports avec leur employeur, que nous analysons à présent.

\subsection{Les rapports avec les patients}

La protection des patients constitue l'argument principal avancé par les professionnels dans leur revendication d'une régulation déontologique, à savoir 
la nécessité, pour le bien des patients, que les professionnels obéissent à des règles qui pèsent sur leurs pratiques quotidiennes, dans le but de garantir la qualité de la prise en charge. On comprend, dans ce contexte, que le code de déontologie reprenne, dans son article 40, le principe traditionnel "primum non nocere", en affirmant que "le médecin doit s'interdire, dans les investigations et interventions qu'il pratique comme dans les thérapeutiques qu'il prescrit, de faire courir au patient un risque injustifié", et dans le même registre, son article 39 prévoit que "les médecins ne peuvent proposer aux malades ou à leur entourage comme salutaire ou sans danger un remède ou un procédé illusoire ou insuffisamment éprouvé. Toute pratique de charlatanisme est interdite". Plus largement, l'article 2 du code de déontologie prévoit que "le médecin, au service de l'individu et de la santé publique, exerce sa mission dans le respect de la vie humaine, de la personne et de sa dignité. Le respect dû à la personne ne cesse pas de s'imposer après la mort".

Certains principes relatifs aux relations entre les médecins et leurs patients ont cependant été mis en débat, en 2002 , lors de la discussion de la loi $n^{\circ 2002-~}$ 303 relative aux droits des patients. L'article 35 alinéa 2 du code de déontologie médicale prévoyait, en effet, jusqu'à cette date, que le médecin pouvait ne pas informer le patient "en cas de pronostic ou de diagnostic grave", situation qu'il "appréciait en conscience". Une telle construction revenait à dire que la décision d'informer ou de ne pas informer le patient revenait au médecin, sous le seul contrôle de sa conscience, règle inconciliable avec le droit du patient à être informé (sauf s'il ne le désire pas), revendication portée, quant à elle, par les associations de patients dans les années 1990. La loi consacrant alors le droit du patient à l'information, le code de déontologie a été tacitement abrogé sur ce point. Mais un tel débat permet de montrer que si le code de déontologie est bien construit pour défendre l'intérêt des patients, il s'agit de l'intérêt des patients dans la conception que s'en font les médecins, et non dans celle des patients eux-mêmes, l'exemple précité permettant de constater que les conceptions en la matière peuvent diverger.

\section{2. Les rapports avec les employeurs}

L'une des questions marquantes de l'histoire de la construction de la déontologie médicale est celle de l'indépendance des professionnels, le principe de l'indépendance étant fortement affirmé dès les années 1920, dans le but d'échapper à un éventuel pouvoir de direction des employeurs quant aux pratiques médicales. C'est la raison pour laquelle l'article 5 du code de déontologie dispose que "le médecin ne peut aliéner son indépendance professionnelle sous quelque forme que ce soit”. En ce qui concerne l'exercice salarié de la médecine, l'article 95 du code décline le principe d'indépendance en prévoyant que "le fait pour un médecin d'être lié dans son exercice professionnel par un contrat ou un statut à un autre médecin, une administration, une collectivité ou tout autre organisme public ou privé n'enlève rien à ses devoirs professionnels et en particulier 
à ses obligations concernant le secret professionnel et l'indépendance de ses décisions", son second alinéa prévoyant quant à lui qu' "en aucune circonstance, le médecin ne peut accepter de limitation à son indépendance dans son exercice médical de la part du médecin, de l'entreprise ou de l'organisme qui l'emploie. II doit toujours agir, en priorité, dans l'intérêt de la santé publique et dans l'intérêt des personnes et de leur sécurité au sein des entreprises ou des collectivités où il exerce". Dans le même registre, l'article 97 ajoute qu'un "médecin salarié ne peut, en aucun cas, accepter une rémunération fondée sur des normes de productivité, de rendement horaire ou toute autre disposition qui auraient pour conséquence une limitation ou un abandon de son indépendance ou une atteinte à la qualité des soins". II faut comprendre ici que, quel que soit le cadre juridique dans lequel le médecin est amené à exercer, le principe d'indépendance relatif aux décisions médicales trouve à s'appliquer. On pourrait cependant rétorquer que ce que dit la déontologie ne trouve pas forcément à s'appliquer en pratique, du fait de la portée limitée de cette dernière, qui ne prend place que dans un décret en Conseil d'État. Rappelons, cependant, comme nous l'avons expliqué à propos des conséquences de l'intégration de la déontologie au droit de l'État, que les lois qui interviennent dans le domaine de la déontologie renvoient systématiquement à cette dernière dès lors que l'on se trouve dans son champ d'application.

On a compris, dans les développements qui précèdent, que ni la manière dont les règles déontologiques sont élaborées, ni leurs objets, ne sont à l'abri des critiques. Nous allons à présent développer cette idée, dans une troisième partie, à propos de questions particulières, cherchant à montrer que la déontologie constitue de facto un instrument de défense des conceptions "traditionnelles" de la médecine.

\section{LA DEONTOLOGIE, INSTRUMENT DE DEFENSE DES CONCEPTIONS TRADITIONNELLES DE LA MEDECINE ?}

Selon le code de la santé publique (article L. 4121-1), l'ordre des médecins "groupe obligatoirement tous les médecins (...) habilités à exercer". Il est, dans ce contexte, chargé de "veille(r) au maintien des principes de moralité, de probité, de compétence et de dévouement indispensables à l'exercice de la médecine (...) et à l'observation, par tous (ses) membres, des devoirs professionnels, ainsi que des règles édictées par le code de déontologie prévu à l'article L. 4127-1", l'article L. 4121-2 ajoutant qu'il "assure la défense de l'honneur et de l'indépendance de la profession médicale”. L'ensemble de ces énoncés, qui concerne le maintien, l'observation des devoirs et des règles de déontologie ainsi que la défense de l'honneur et de l'indépendance, renvoie bien à la défense d'un certain nombre de valeurs et de conception de la médecine que l'on peut qualifier de traditionnelles dans la mesure où elles figurent dans la loi depuis 1945, répondant à une 
revendication exprimée par les médecins dès 1845 . Tel est d'ailleurs l'une des critiques récurrentes adressées à l'ordre des médecins (notamment en ce qui concerne sa résistance à certaines évolutions sociales comme la contraception dans les années 1960 ou l'interruption volontaire de grossesse dans les années 1970): il serait conservateur, voire réactionnaire. Si le deuxième épithète peut être discuté (outre la critique idéologique qu'il comporte), le premier ne saurait étonner le juriste dans la mesure où l'ordre professionnel est justement conçu pour poursuivre une telle action conservatrice.

On comprend alors que la déontologie, préparée par l'ordre, et dont il doit assurer le respect des règles, s'inscrive dans une même logique. Nous allons le montrer à propos de trois questions: la défense de la médecine libérale (1), le paternalisme médical (2) et l'indépendance professionnelle (3).

\section{La médecine libérale}

Comme nous l'avons exposé dans la première partie, les règles des quatre codes de déontologie qui se sont sucédés depuis 1947 se fondent sur des principes du règlement intérieur du conseil national de l'ordre de 1941, qui reprend lui-même les principes de la "charte médicale" de 1927. Or, il est important de connaître les enjeux de cette production, ceux-ci ayant laissé une trace durable dans le code de déontologie (et donc dans l'ensemble du droit français). Le contexte dans lequel la charte est rédigée et celui de l'essor de la médecine sociale et de la préparation d'une loi sur les sociétés de secours mutuel, c'est-à-dire les organismes d'assurance mutuelle destinés à prendre en charge le coût des soins. Un syndicat minoritaire de médecins, opposé à l'essor de la médecine sociale, va élaborer la charte et réussir, après quelques péripéties politiques, à en faire accepter les principes par le gouvernement. La charte de la médecine libérale contient ainsi cinq principes qui concernent le contenu de la déontologie: la liberté de choix du médecin par le patient; le secret professionnel; le droit de recevoir des honoraires pour chaque patient traité; "l'entente directe" entre le professionnel et le patient, c'est à dire le principe selon lequel le patient paye directement le médecin, sans passer par un tiers (le patient pouvant ensuite se faire rembourser); la liberté thérapeutique (dont la liberté de prescription) du médecin. On l'aura compris, de tels principes empêchaient - et avaient bien pour but d'empêcher -, à l'époque, la possibilité d'organiser l'exercice salarié de la médecine (notamment le paiement à l'acte et l'entente - ainsi que le paiement - directs). II faudra attendre plus de 30 ans, et une loi, pour que celui-ci devienne possible. Mais aujourd'hui encore, selon l'article L. 162-2 du code de la sécurité sociale, qui pose les principes fondamentaux gouvernant les rapports entre les médecins et l'assurance maladie (l'assurance obligatoire relative aux soins) "dans l'intérêt des assurés sociaux et de la santé publique, le respect de la liberté d'exercice et de l'indépendance professionnelle et morale des médecins est assuré conformément aux principes déontologiques fondamentaux que sont 
le libre choix du médecin par le malade, la liberté de prescription du médecin, le secret professionnel, le paiement direct des honoraires par le malade, la liberté d'installation du médecin".

La déontologie a donc constitué la matrice - et contient toujours les principes - défendant une certaine conception de la médecine libérale. Soulignons cependant que certains de ces principes sont aujourd'hui largement amendés notamment par le droit de l'assurance-maladie. Dans une telle hypothèse, les règles déontologiques restent applicables, mais dans la mesure où plus de 99 $\%$ des médecins sont conventionnés (les règles relatives aux relations entre l'assurance-maladie et les médecins font l'objet de "conventions" conclues entre les organismes d'assurance-maladie et les syndicats professionnels), l'exception devient, statistiquement, la règle. C'est ainsi, par exemple, qu'on a vu se développer, notamment pour les patients les plus pauvres, le système du "tiers payant", qui constitue une dérogation au principe de "l'entente directe".

\section{Le paternalisme médical}

La doctrine dite du "paternalisme médical" a été théorisée dans les années 1950, par le président du conseil national de l'ordre des médecins, Louis Portes, qui écrivait: 'face au patient, inerte et passif, le médecin n'a en aucune façon le sentiment d'avoir affaire à un être libre, à un égal, à un pair, qu'il puisse instruire véritablement. Tout patient est, et doit être pour lui, comme un enfant à apprivoiser, non certes à tromper - un enfant à consoler, non à abuser - un enfant à sauver, ou simplement à guérir, à travers l'inconnue des péripéties". Cette doctrine aboutissait à conférer au médecin un véritable "droit de guérir", le patient devant se contenter d'obéir au médecin pour assurer sa guérison. Une question illustrative de cette conception est celle de l'observance de la prescription médicale. Ainsi, le code de déontologie médicale du 27 juin 1947 disposait, dans son article 30, qu' 'après avoir établi un diagnostic ferme comportant une décision sérieuse, surtout si la vie du malade est en danger, un médecin doit s'efforcer d'imposer l'exécution de sa décision" (nous soulignons). Le code du 28 novembre 1955 prévoyait quant à lui en son article 29 que le praticien devait "s'efforcer d'obtenir l'exécution du traitement prescrit". Le code de déontologie du 28 juin 1979 prévoyait, en outre, dans son article 37, que le médecin devait "s'efforcer d'obtenir la bonne exécution du traitement". Le code du 6 septembre 1995 prévoit, enfin, que "Le médecin doit formuler ses prescriptions avec toute la clarté indispensable, veiller à leur compréhension par le patient et son entourage et s'efforcer d'en obtenir la bonne exécution". On constate que si les formulations ont évolué pour perdre en vigueur, la logique sous-jacente reste la même: le patient doit obéir à la prescription.

Cette conception "impérialiste" de l'exercice médical - même si elle a progressivement été amendée jusque dans les années 2000 -, niant au patient 
la capacité à décider dans le cadre de cette relation, a amené la profession médicale - ou tout au moins son ordre professionnel - à s'opposer, jusqu'en 2002, à l'évolution des règles relatives à l'information du patient. Jusqu'à la loi n²002-303-du 4 mars 2002, en effet, l'ordre des médecins était à l'origine des règles en matière d'information des patients, celles-ci étant contenues dans l'article 35 du code de déontologie, dont la modification éventuelle renvoyait, selon l'article L. 4121-7 du code de la santé publique, à son initiative. On aurait alors pu imaginer que le code de déontologie médicale évolue, à l'initiative de l'ordre des médecins, pour correspondre à l'évolution des normes sociales en la matière, telle qu'en attestent les règles qui résultent de la loi relative aux droits des patients, auquel cas ce pan de la réforme eût été techniquement inutile. Faute d'une telle initiative, l'ordre des médecins a amené le législateur à intervenir dans un domaine relevant traditionnellement de la régulation déontologique. La réglementation de la question de l'information des patients va même finir d'échapper à la régulation déontologique puisque l'article L. 1111-2 alinéa 6 du Code de la santé publique prévoit que "des recommandations de bonnes pratiques sur la délivrance de l'information sont établies par l'Agence nationale d'accréditation et d'évaluation en santé et homologuées par arrêté du ministre chargé de la santé". Avant la loi du 4 mars 2002, les médecins avaient donc déontologiquement le choix d'informer ou non leurs patients en cas de pronostic ou diagnostic grave, ne rendant de comptes qu'à leur conscience; depuis le 4 mars 2002, ils ont le devoir d'informer leurs patients qui disposent, désormais, d'un véritable droit subjectif à être informés. La déontologie, longtemps réceptacle des règles relatives à l'information des patients, doit donc aujourd'hui se contenter de suivre la loi, précisée par des recommandations de bonne pratique.

Un enseignement plus général peut, en outre, en être de tiré: la déontologie ne couvre plus, depuis 2002, l'ensemble des comportements professionnels. D'autres questions, traditionnellement déontologiques ont également depuis cette date, fait l'objet d'une intervention légale: l'encadrement de la fin de vie (2005), l'information sur les honoraires (2007) et la discrimination à l'encontre des patients les plus pauvres (2010).

\section{L'indépendance professionnelle}

L'un des soubassements traditionnels de la déontologie réside dans l'indépendance du professionnel. L'idée fondamentale est ici que, pour être au service du patient, le professionnel doit être indépendant de tout autre pouvoir. L'orientation effective de l'activité dans l'intérêt du patient est alors renvoyée à la conscience du professionnel; une formule traditionnelle pour résumer la relation médicale est alors la suivante: "la rencontre entre une conscience et une confiance". Le principe de l'indépendance professionnelle est ainsi inscrit au fronton du code de déontologie, dans l'un de ses premiers articles, l'article 5 , selon lequel "le médecin ne peut aliéner son indépendance professionnelle 
sous quelque forme que ce soit", et décliné dans d'autres articles, notamment l'article 95 précité relatif à l'exercice salarié de la médecine selon lequel "le fait pour un médecin d'être lié dans son exercice professionnel par un contrat ou un statut à un autre médecin, une administration, une collectivité ou tout autre organisme public ou privé n'enlève rien à ses devoirs professionnels et en particulier à ses obligations concernant le secret professionnel et l'indépendance de ses décisions". Et cette exigence est relayée, au-delà du code de déontologie, par la loi, et notamment le code de la santé publique à propos des médecins agents publics, selon lequel "le directeur (de l'hôpital) exerce son autorité sur l'ensemble du personnel dans le respect des règles déontologiques ou professionnelles (...) et de l'indépendance professionnelle du praticien dans l'exercice de son art".

En outre, certaines questions déontologiques traditionnelles, qui n'ont pas explicitement pour objet l'indépendance, peuvent être analysées comme la défendant indirectement: tel est le cas du secret professionnel. Le discours des professionnels est, à ce propos, emprunt de fermeté, faisant même remonter, pour le légitimer, le principe du secret à Hippocrate (oubliant, au passage, une éclipse de près de 2000 ans d'histoire qui ne connait pas le secret, de l'Empire Romain au XVIIle siècle...). En France, le secret professionnel, intégré au code pénal en 1810, est destiné à protéger la vie privée des personnes; il est repris, depuis le XIXe siècle, dans les différentes déontologies. Mais si son intérêt est indéniable pour les patients, le secret constitue également un gage d'indépendance pour les professionnels: il leur permet, en effet, de se soustraire à une demande d'informations relative à leur activité, faisant alors échapper cette dernière au regard d'autrui, rendant ainsi son contrôle difficile.

Mais parallèlement, les médecins ont constamment revendiqué la possibilité de se dispenser du secret dès lors que la discussion se déroule entre eux, et de fortes présomptions existent pour penser qu'ils s'en dispensaient, de facto, mais illégalement, avant la loi du 4 mars 2002, dès lors qu'ils estimaient qu'il en allait de l'intérêt du patient (mais, encore une fois, dans leur propre conception de cet intérêt...). II s'agissait, en tous cas, d'une forte revendication professionnelle à laquelle a répondu, et répond, depuis cette date, l'article L. 1110-4 du code de la santé publique selon lequel, certes, "toute personne prise en charge (...) a droit au respect de sa vie privée et du secret des informations la concernant", mais également selon lequel "deux ou plusieurs professionnels de santé peuvent toutefois, sauf opposition de la personne dûment avertie, échanger des informations relatives à une même personne prise en charge, afin d'assurer la continuité des soins ou de déterminer la meilleure prise en charge sanitaire possible".

On peut constater ici que, face aux règles du code pénal visant à limiter l'indépendance des médecins en leur imposant le secret, les professionnels ont revendiqué, et obtenu, une dérogation au principe général allant dans le sens de leur indépendance, et qui recouvre, de fait, la quasi totalité des discussions professionnelles: les professionnels peuvent échanger, à propos d'un patient, avec 
d'autres professionnels dans l'exercice de sa profession (certes afin d'assurer la continuité des soins ou de déterminer la meilleure prise en charge sanitaire possible...), ce qui correspond à leur revendication. Le secret reste, en revanche, de principe en ce qui concerne les relations avec les non professionnels, ce qui correspond à leur intérêt. Certes, on peut affirmer qu'il en va également de l'intérêt du patient, mais force est de constater que le système ne contrarie aucunement celui des professionnels. On pourrait également objecter qu'il s'agit ici d'un débat relatif à des normes légales; tel est effectivement techniquement le cas, mais il faut bien comprendre que pour les professionnels, et pour leurs relais au Parlement, la question est principalement déontologique et ne constitue que secondairement une question légale.

D'autres questions pourraient être analysées de même: encadrement de la fin de vie, gestion des discriminations des patients pauvres, détermination des honoraires par exemple. On se souvient en outre que la même logique d'indépendance était à l'œuvre en ce qui concerne l'exercice libéral de la médecine ainsi que le paternalisme médical analysés dans les subdivisions précédentes. La défense de l'indépendance des professionnels constitue donc un principe déontologique fondamental, et peut-être même le principe déontologique fondamental, irrigant l'ensemble de la déontologie.

\section{CONCLUSION}

On peut, en conclusion, résumer un certain nombre des éléments précédents et les mettre en perspective.

On peut ainsi affirmer que le code de déontologie médicale constitue une norme fondamentale de l'encadrement de l'exercice de la médecine. Jusqu'à une période récente, il constituait même l'instrument exclusif de cet ordonnancement, l'ensemble des règles relatives aux relations professionnelles des médecins prenant place en son sein. On pouvait alors considérer que le code de déontologie médicale constituait un véritable "code d'exercice professionnel". Cette affirmation doit aujourd'hui être nuancée: depuis la loi du 4 mars 2002 relative aux droits des patients, une partie des règles relatives au rapport entre ces derniers et les médecins trouve son siège dans le code de la santé publique. La déontologie ne couvre donc plus, depuis 2002, l'ensemble des comportements professionnels. Ce faisant, la loi du 4 mars 2002 constitue une indéniable avancée démocratique, mettant fin à une hypocrisie institutionnelle, la préparation des codes de déontologie par les professionnels laissant croire que ces règles ne concernaient effectivement que les professionnels alors qu'un certain nombre d'entre elles notamment celles relatives à l'information ou consentement des patients ainsi qu'à l'indépendance des professionnels et donc, indirectement, au pouvoir de leurs employeurs en ce qui les concerne figuraient ou figurent dans le code de déontologie. 
Leur contenu peut, quant à lui, être présenté en référence à deux fonctions assurées par le code de déontologie médicale: l'analyse montre, en effet, qu'il constitue, d'une part un instrument de contrôle de la profession médicale sur les professionnels, d'autre part un instrument de l'indépendance des professionnels vis-à-vis de leurs autres interlocuteurs.

Le contrôle de la profession médicale sur les médecins par l'intermédiaire du code de déontologie résulte, d'une part, des possibilités de contrôle de l'ordre sur les contrats professionnels (les contrats doivent rappelons-le, être transmis à l'ordre qui vérifie leur absence de contrariété à la déontologie), d'autre part de la possibilité, pour l'ordre professionnel, de sanctionner disciplinairement un professionnel qui manquerait à la déontologie. L'indépendance professionnelle est, quant à elle, assurée par les dispositions du code de déontologie qui la reconnaissent, auxquelles la loi renvoie systématiquement, et qui constitue la norme de référence en ce qui concerne les relations du médecin avec l'ensemble de ses interlocuteurs.

Le code de déontologie constitue donc, in fine, une construction technique qui renvoie aux valeurs et aux conceptions de la profession médicale, et qui permet de les imposer, d'une part aux professionnels qui leur seraient récalcitrants, d'autre part aux non professionnels. Et il a fallu attendre 2002 pour que le législateur contrarie, en ce qui concerne la question ô combien sensible de l'information du patient, les conceptions professionnelles en la matière. Encore, si les médecins avaient choisi d'aligner les dispositions du code de déontologie sur les conceptions sociales des patients et non sur les leurs, les règles en la matière resterait-elle aujourd'hui dans le code de déontologie. Mais ils ne l'ont justement pas fait.

\section{BIBLIOGRAPHIE}

ALMERAS, Jean-Pierre; PÉQUIGNOT, Henri. La déontologie médicale. Paris: Litec, 1996.

BENTHAM, Jeremy. Déontologie ou science de la morale. Paris: Charpentier, 1834. $2 \mathrm{~V}$.

FRANCE. Code de déontologie médicale. Disponible en: <http://www.conseilnational.medecin.fr/groupe/17/tous>. Acèss: 2 juillet 2012.

FRANCE. Code de la santé publique. Disponible en: <http://www.legifrance. gouv.fr/affichCode.do?cidTexte=LEGITEXT000006072665\&dateTex te=20120703 $>$. Acèss: 2 juillet 2012 .

HATZFELD, Henri. Le grand tournant de la médecine libérale. Paris: Éditions Ouvrières, 1963. 
JAUNAIT, Alexandre. Comment pense l'institution médicale? Paris: Dalloz, 2005. (Nouvelle Bibliothèque de Thèses).

LEONARD, Jacques. La médecine entre les pouvoirs et les savoirs: histoire intellectuelle et politique de la médecine au XIX ${ }^{e}$ siècle. Paris: AubierMontaigne, 1981.

MORET-BAILLY, Joël. Les déontologies. Aix-en-Provence: Presses Universitaires d'Aix-Marseille, 2001.

ORDRE NATIONAL DES MEDECINS. Commentaire du code de déontologie. Disponible en: <http://www.conseil-national.medecin.fr/groupe/17/tous>. Acèss: 22 mai 2012.

PORTES, Louis. A la recherche d'une éthique médicale. Paris: Masson, 1954.

RODWIN, Marc. Conflicts of interest and the future of medicine. Oxford: Oxford University Press, 2011.

SIMON, Maxime. Déontologie médicale ou des droits et des devoirs des médecins dans l'état actuel de la civilisation. Paris: Baillière, 1845.

THOUVENIN, Dominique. Le secret médical et l'information du malade. Lyon: Presses Universitaires de Lyon, 1982. 